\title{
Philosophiques
}

\section{La science-fiction et les héroïnes de la modernité}

\section{Élisabeth Vonarburg}

Volume 21, numéro 2, automne 1994

Les femmes et la société nouvelle

URI : https://id.erudit.org/iderudit/027286ar

DOI : https://doi.org/10.7202/027286ar

Aller au sommaire du numéro

Éditeur(s)

Société de philosophie du Québec

ISSN

0316-2923 (imprimé)

1492-1391 (numérique)

Découvrir la revue

Citer ce document

Vonarburg, É. (1994). La science-fiction et les héroïnes de la modernité.

Philosophiques, 21(2), 453-457. https://doi.org/10.7202/027286ar

\section{Résumé de l'article}

Il y a une convergence obligée entre SF et féminisme. D'abord la SF a 'pour ancêtre l'utopie, et imagine donc des modèles de société autres, tout comme le féminisme est obligé de le faire; ensuite, la SF permet d'aborder les problèmes des femmes d'un point de vue créatif et non réactif comme la littérature normative; enfin la distance mythique retrouvée dans la SF permet aux auteures et lectrices d'accéder pleinement au registre héroïque, qui leur est souvent dénié par la littérature normative. 


\title{
La science-fiction et les hérönes de la modernité
}

\author{
par \\ Elisabeth Vonarburg
}

RESUME : Il y a une convergence obligée entre SF et féminisme. D'abord la SF a pour ancêtre l'utopie, et imagine donc des modèles de société autres, tout comme le féminisme est obligé de le faire; ensuite, la SF permet d'aborder les problèmes des femmes d'un point de vue créatif et non réactif comme la littérature normative; enfin la distance mythique retrouvée dans la SF permet aux auteures et lectrices d'accéder pleinement au registre héroïque, qui leur est souvent dénié par la littérature normative.

ABSTRACT : There is a necessary convergence between science fiction and feminism. On one hand, SF has utopia for an ancestor and therefore invents other societal models, as feminism itself must do. On the other hand, SF deals creatively with women's problems, not just reactively like mainstream literature does. And finally, due to the mythic perspective that permeates $S F$, writers and readers can fully participate in the heroic, which is often denied to by mainstream literature.

Je vous demanderai d'admettre sans démonstration excessive trois points qui me paraissent fondamentaux.

D'abord, que la pensée féministe est de nature fondamentalement utopiste : une fois conscience prise et compte tenu des pépins de la réalité, nous sommes obligées d'imaginer l'autrement autre part qu'ici, et le premier autre part, c'est le nulle part de l'utopie (outopia), qui est aussi une «bonne part » (eutopia), comme ça tombe bien ! Ensuite, que l'utopie est l'une des grands-mères de la science-fiction : toutes deux portent sur le présent lou « le réel », synonymie abusive selon moi) le même regard en biais, qui constitue la même expérience mentale, la même critique des préjugés, et qui conduit à la même construction de modèles de sociétés, parallèles ou perpendiculaires, mais différentes. Et donc (et enfin), que la science-fiction constitue un véhicule d'une extrême richesse potentielle pour la pensée féministe, laquelle malheureusement, d'après ce que j'ai entendu ici jusqu'à présent, ne semble pas tellement la connaître et c'est bien dommage.

La convergence obligée de la science-fiction et de la pensée féministe se fait d'abord, pour moi, dans la mesure où la SF moderne écrite par les femmes 
a revivifié le modèle traditionnel, statique, de l'utopie en des utopies « ambiguës »'. c'est-à-dire ayant partie liée avec le temps et le changement.

Elle se fait ensuite dans la mesure où la SF offre à l'imaginaire d'autres chronotopes, c'est-à-dire comme l'indique le néologisme, d'autres temps \& lieux contextuels que les contraignants Ici-\&-maintenant ou Ailleurs-छ-hier de la littérature non science-fictive où nous sommes condamnées aux modes réactifs de la déploration, de la constatation ou de la révolte, le « terre à terre » des femmes dont parlait Nicole Brossard - modes nécessaires, légitimes, mais dont l'inévitabilité a quelque chose d'un peu déprimant, d'un peu monotone et de fort peu propice aux modes véritablement créatifs de l":maginaire. Seule la poésie permet de déborder, ou le « réalisme magique » : tous deux sont «dans les marges ». Mais tout le monde ne peut être poète, et des rapports individuels différents au temps et à l'espace du langage exigent d'autres types de création. En ce qui me concerne, (car je vais abandonner le « on » du discours pseudoobjectif; mon «je », même s'il est problématique, j’y tiens !), en ce qui me concerne, donc, c'est la fiction narrative, et c'est surtout la science-fiction, parce qu'elle aussi se situe dans des marges; de ce point de vue, je serais presque prête à accepter la ridicule étiquette de « para-littéraire », parce que l'à-côté de la SF, revendiqué ou imposé, constitue indéniablement un espace de liberté par rapport à la littérature normative.

La SF me permet de faire jouer divers personnages dans divers modèles de sociétés afin d'éclairer et critiquer (et non fuir et ignorer) les/mes situations actuelles. Mais elle va plus loin en me permettant d'essayer d'imaginer d'autres modèles, et pour cela d'autres façons de poser les questions qui sont, ou devraient selon moi être, à la base de toute pensée féministe : « qu'est-ce qu'être femme? », par exemple, lou, si on l'exprime en termes science-fictifs, « qu'est-ce qu'être la moitié femelle de l'espèce dimorphique qui habite la planète Terre? »- une optique un peu différente...). Qu'est-ce, corrélativement, qu'être homme? Et, la question fondamentale de la SF : « qu'est-ce qu'un être humain? » La SF est une littérature qui dispose de tous les outils de la connaissance scientifique pour essayer de répondre à ces questions - tout comme la pensée féministe, pour y répondre aussi, se voit obligée de mettre en question et à contribution tout le champ de la connaissance, aussi bien la philosophie que la philologie, la biologie que la sociologie : une convergence de plus.

Compagne de route de la pensée féministe, donc, il ne fait pour moi aucun doute que la SF l'est (compagnonnage d'autant plus réjouissant que la SF est encore un man's land, aussi bien dans sa production que dans sa consommation et dans l'image que s'en fait le public non prévenu). Elle est donc moderne, cela ne fait pas un pli. Est-elle hérö̈que, puisque après tout c'était dans ces

I. Cest le sous-titre du roman... matriciel de Ursula Le Guin. La Main gauche de la nuit, Laffont, Paris, 1971. 
alentours que devait se situer ma communication initiale, et quej'ai maintenu des « héroïnes » dans mon titre?

Oui, et c'est une autre raison pour moi d'en écrire, qui vient de plus loin. $\checkmark$ ai toujours voulu être un héros - mais j'ai toujours su que je n'étais pas un garçon (les choses étant ce qu'elles étaient à l'époque, et sont encore, j'ai pu le regretter parfois, mais je me suis soignée !). Le fait est que mes premières lectures, mes traumatismes littéraires d'enfance, en quelque sorte, furent les mythes, contes et légendes de tous les temps et de tous les pays. Lecture fascinante, mais déconcertante pour une petite fille : pratiquement tous les héros sont des hommes, et les personnages féminins, eh bien... Mais, comme presque toutes les petites filles, je suis rentrée bon gré mal gré dans le moule de l'identification obligée (et frustrante) au masculin, je me suis adaptée à cette norme, quasi modo, sans avoir plus conscience que cela, je l'avoue, d'un quelconque problème identitaire. Le transvestisme s'est poursuivi quand je suis entrée dans la littérature « pour adultes », et je suis tombée en amour avec Hugo, Baudelaire, Rimbaud, Dostoïevski et Camus sans problème majeur de conscience...

Mais c'était le milieu des années ig6o, et même dans ma petite province française bien endormie, quelque chose bouillonnait. Ce fut la découverte de la science-fiction qui me permit de laisser sortir ma vapeur : non, ce n'était pas vrai que « c'est comme ça, ça a toujours été comme ça, ce sera toujours comme ça »! L'univers et corrélativement l'être humain étaient bien plus complexes, plus mystérieux, plus merveilleux qu'on n'avait voulu me le faire croire... Et surtout, les récits de SF me permettaient de renouer avec l'héroïsme dans un registre satisfaisant pour mon imaginaire.

Car enfin, les héros au sens mythique n'ont plus cours dans la littérature moderne. La notion même de personnage s'est tellement fait étriller depuis 40 ou 50 ans qu'on ose à peine baptiser ainsi cet ensemble aléatoire de mots sur une feuille, ou cet ensemble de fonctions dans la Mécanique du Texte... Mais dans la SF, grâce au décalage spatio-temporel essentiel au genre, des figures symboliques puissantes peuvent (rélapparaître, et leur degré d'efficace, dans l'imaginaire, est renforcé et multiplié par le système idéologique qui les valide - par le mythe, n'hésitons pas à employer le mot, de la science et de ses possibles. Et devant ces figures familières, on peut soudain envisager d'autres postures. J'ai retrouvé mes dragons, mes magiciens, mes immortels, mes doubles... mais sans la surnature qui les éloignait trop de moi : l'extra-terrestre est un dragon intelligent, avec qui échanger et non plus seulement se battre, le magicien est un savant ou un mutant, l'immortel est un voyageur perdu dans le temps, le double n'est plus une menace mais un enrichissement...

Hélas, ma joie fut de courte durée. Des figures héroïques, dans la SF, il y en avait, certes. Toutes des hommes. Encore. Les héroïnes, comme partout ailleurs, étaient des Vierges, jolies petites choses fragiles et stupides qui se tordaient les mains ou l'équivalent au sommet d'une tour ou l'équivalent. Ou encore, comme partout ailleurs, des Garces momentanément dangereuses mais ultimement punies... Ou au mieux (au pire?) des Mères-épouses 
méritantes qui s'employaient à élever leur petite famille nucléaire selon la sacro-sainte loi de la séparation des tâches, quelque part sur Aldébaran XVIII, en l'an de grâce 3269 et demi.

Bref, le point aveugle de la SF quant aux figures et positions sexuales était si vaste qu'on aurait pu y enfourner le système solaire tout entier.

Mais heureusement c'était la fin des années ig6o, et la révolution féministe se fit sentir aussi dans la SF : les femmes y entrèrent en force, et changèrent à jamais le visage du genre. Certes, les chercheuses et théoriciennes littéraires sont encore un peu timides à revendiquer le terme « héroïne », si terriblement dévalué ${ }^{2}$, mais les écrivaines sont, comme d'habitude, plus en avance... Dans les dix dernières années, cependant, il faut reconnaître qu'il y a eu de la part de plusieurs chercheuses féministes des efforts méritoires pour donner à la notion d'héroïsme féminin ses lettres de noblesse, surtout en étudiant de quelle façon il se distingue littérairement de l'héroüsme masculin jusque-là considéré comme la norme ${ }^{3}$. Il existe bel et bien une héroüne moderne, dont le trajet ne suit pas tout à fait la norme de Joseph Campbell ${ }^{4}$; rappelons pour mémoire quelques descriptions de celui-ci :

Le pouvoir arraché à l'ennemi Ipar le héros], la liberté arrachée à la malignité du monstre, l'énergie vitale libérée des anneaux étouffants du tyran Grippedur - c'est une femme qui les symbolise. Si lle hérosl est le roi du monde, elle est le monde, et s'il est le héros elle est la gloire. La femme est l'image de sa destinée, qu'il doit libérer des circonstances où elle est enfouie ${ }^{5}$.

Ou encore, à propos de l'accession des adolescents à l'âge adulte :

Un nouvel élément de rivalité entre dans le tableau : le fils contre le père pour la maîtrise de l'univers, et la fille contre la mère pour être le monde qui sera maîtrisé ${ }^{6}$.

Eh bien, pas vraiment. Et la dédaigneuse norme freudienne ne s'applique pas forcément non plus à la pulsion héroïque féminine, le « vouloir être hérö̈que, chez une femme, ce n'est que l'envie du pénis $\gg . .$.

Heureusement, donc, grâce au travail révolutionnaire et libérateur des écrivaines de science-fiction,j'ai pu enfin trouver de vraies héroïnes dans ce que

2. On parle chez les Anglo-Saxonnes de « female hero »...

3. Voir en particulier Carol Pearson et Katherine Pope, The Female Hero in American and British Literature, New York et Londres, Bowker, 1981.

4. He Hero with a Thousand Faces, New York, Princeton Bollingen Press, troisième réédition, 1973. L'original date de 1949 .

5. «The hegemony wrested from the enemy, the freedom wrested from the malice of the monster, the life energy released from the coils of the tyrant Holdfast - is symbolized by a woman - if his stature is that of the world monarch, she is the world, and if he is the warrior, she is fame. She is the image of his destiny which he is to release from the pressure of enveloping circumstances. 》

6. «There is a new element of rivalry in the picture : the son against the father for the mastery of the universe, and the daughter against the mother to be the mastered world. » 
je lisais, et surtout imaginer les miennes. La pulsion héroïque est pour moi inséparable de la pulsion mythique; toute personne humaine a besoin de se relier au monde par des histoires qui la définissent et la légitiment comme hérö̈ne symbolique de sa propre existence - et en tant que femme, j'ai besoin d'autres versions des mythes existants, sinon de mythes nouveaux! Je ne sais si l'on peut véritablement créer des mythes nouveaux, mais je sais qu'en tant que femme, le simple fait d'essayer de créer les miens est enrichissant et formateur, même dans l'échec, car cet échec m'oblige alors à me poser d'autres questions, (ou les mêmes autrement...), à tenter dans et par l'écriture d'autres expériences intellectuelles, affectives et spirituelles, élargissant ainsi (aux deux sens du terme !) mon imaginaire, mon être même.

Sans doute n'aurais-je jamais vraiment écrit si je n'avais pas rencontré la science-fiction. Il existe, a-t-on dit $^{7}$, un rapport imaginaire au monde qui en est un de conquête et de domination par la re-création de ce qui est - celui des Balzac et autres Dickens, les « réalistes ». Il en serait un autre dont la pulsion fondamentale est toujours celle de l'appropriation, mais où l'on désire s'approprier en imaginant ce qui n'est pas; c'est le mode des « fantastiqueurs », je crois. Le mode de la SF, et le mien, quelque part entre les deux, ou dans leurs marges respectives, serait de s'approprier ce qui est, comme les « réalistes », mais en le métamorphosant profondément - comme les « fantastiqueurs ».

Et il se trouve que c'est aussi, je le crois fermement, la seule issue possible pour une femme. 